ARTICLE

https://doi.org/10.1038/s41467-019-11372-w

\title{
Local orbital degeneracy lifting as a precursor to an orbital-selective Peierls transition
}

\author{
E.S. Bozin (1) 1, W.G. Yin (1) 1, R.J. Koch', M. Abeykoon², Y.S. Hor ${ }^{3,5}$, H. Zheng ${ }^{3}$, H.C. Lei ${ }^{1,6}$, C. Petrovic ${ }^{1}$, \\ J.F. Mitchell ${ }^{3} \&$ S.J.L. Billinge ${ }^{1,4}$
}

Fundamental electronic principles underlying all transition metal compounds are the symmetry and filling of the $d$-electron orbitals and the influence of this filling on structural configurations and responses. Here we use a sensitive local structural technique, x-ray atomic pair distribution function analysis, to reveal the presence of fluctuating local-structural distortions at high temperature in one such compound, $\mathrm{Culr}_{2} \mathrm{~S}_{4}$. We show that this hitherto overlooked fluctuating symmetry-lowering is electronic in origin and will modify the energylevel spectrum and electronic and magnetic properties. The explanation is a local, fluctuating, orbital-degeneracy-lifted state. The natural extension of our result would be that this phenomenon is likely to be widespread amongst diverse classes of partially filled nominally degenerate $d$-electron systems, with potentially broad implications for our understanding of their properties.

\footnotetext{
${ }^{1}$ Condensed Matter Physics and Materials Science Department, Brookhaven National Laboratory, Upton, NY 11973, USA. ${ }^{2}$ Photon Sciences Division, Brookhaven National Laboratory, Upton, NY 11973, USA. ${ }^{3}$ Materials Science Division, Argonne National Laboratory, Argonne, IL 60439, USA. ${ }^{4}$ Department of Applied Physics and Applied Mathematics, Columbia University, New York, NY 10027, USA. ${ }^{5}$ Present address: Department of Physics, Missouri University of Science and Technology, Rolla, MO 65409, USA. ${ }^{6}$ Present address: Department of Physics and Beijing Key Laboratory of Opto-electronic Functional Materials and Micro-nano Devices, Renmin University of China, 100872 Beijing, China. Correspondence and requests for materials should be addressed to E.S.B. (email: bozin@bnl.gov) or to S.J.L.B. (email: sb2896@columbia.edu)
} 
B roken symmetry ground states are often found in transition metal systems exhibiting emergent properties such as metal-insulator transitions ${ }^{1-3}$, charge-ordered and chargedensity wave states ${ }^{4}$, colossal magnetoresistive effects ${ }^{5}$, frustrated magnetism $^{6,7}$, pseudogap $^{8}$ and high-temperature superconductivity ${ }^{9,10}$. These are generally driven by electronic interactions understood as Fermi-surface nesting ${ }^{1-13}$, Peierls distortions $^{14,15}$, and cooperative Jahn-Teller effects ${ }^{16}$. These phenomena have energy scales of hundreds to thousands of $\mathrm{meV}^{17}$, corresponding to thousands of Kelvin, yet the broken symmetries tend to appear at much lower temperatures, typically $10^{1}-10^{2} \mathrm{~K}$. The symmetry-broken states at low temperature, have been extensively studied. Fewer details are known about what happens when these materials transition to crystallographically higher symmetry structures upon warming.

Here we present a study that reveals critical insights into the unaccounted for separation in energy scales by applying a quantitative local structure probe, atomic pair distribution function analysis (PDF), to a model material system that exemplifies this behavior. The material system, $\mathrm{CuIr}_{2} \mathrm{~S}_{4}$, has rich broken symmetries in its ground state ${ }^{18}$, including the formation of magnetic singlet Ir-Ir pairs, which disappear on warming through a structural transition that is also, concurrently, a metalinsulator transition (MIT). The PDF analysis reveals difficult to detect but important local-structural distortions that exist at high temperatures, something that has been seen before in other systems (see for example Billinge et al. ${ }^{19}$ ). However, the special scattering characteristics of this system, together with our detailed temperature and doping dependent study with multiple dopant species, exposes fine details of the phenomenon establishing it as a robust but fluctuating $d$-orbital-degeneracy-lifted (ODL) state that is observed to the highest temperatures measured. Much lower elastic energies govern the long-range ordering of the preformed local symmetry-broken ODL objects in these structurally compliant materials ${ }^{20}$ which therefore occurs at temperatures much lower than the electronic energies of ODL formation. Interestingly, in $\mathrm{CuIr}_{2} \mathrm{~S}_{4}$ it is not the formation of the ODL objects, but their ordering that precipitates the MIT and magnetic dimer formation of the ground state. The fluctuating ODL state is stabilized electronically by breaking $d$-electron orbital degeneracies and as such is likely to be a phenomenon that is widespread, though not widely appreciated, among the many materials with incompletely filled $d$-electron manifolds ${ }^{21,22}$, many of which have important emergent low-temperature electronic and magnetic behaviors, from classics such as manganites ${ }^{11,23,24}$, cuprates $^{4,9,25}$, and iron chalcogenides/pnictides $7,10,26-29$, to materials featuring exotic low-temperature orbital molecules ${ }^{30,31}$. It may also explain the unexpected observation of phonon-glass-like thermal conductivity in various transition metal oxides ${ }^{32}$.

\section{Results}

Long-range orbital and charge order and spin dimerization. The low-temperature insulating state in $\mathrm{CuIr}_{2} \mathrm{~S}_{4}{ }^{33-35}$ consists of ordered $\operatorname{Ir}^{3+}\left(5 d^{6}\right)$ and $\operatorname{Ir}^{4+}\left(5 d^{5}\right)$ ions ${ }^{36}$, with a four-fold periodicity, an example of tetrameric charge ordering ${ }^{37}$. Concurrently, spin dimerization of $\operatorname{Ir}^{4+}$ pairs occurs within the tetramer, with large associated structural distortions as they move towards each other, making this charge order particularly amenable to detection using structural probes ${ }^{18}$. Notwithstanding the complexities of the insulating state, including formation of remarkable three-dimensional $\mathrm{Ir}_{8}^{3+} \mathrm{S}_{24}$ and $\mathrm{Ir}_{8}^{4+} \mathrm{S}_{24}$ molecule-like assemblies embedded in the lattice, its quasi-one-dimensional character was unmasked and MIT attributed to an orbitalselective Peierls mechanism, postulated from topological considerations ${ }^{38}$. The global symmetry lowering at the MIT was declared to lift the existing $t_{2 g} d$-orbital degeneracies ${ }^{38}$. Although the high-temperature crystallographically cubic metallic state $e^{18,33}$ appears to be undistorted, with nominally $\operatorname{Ir}^{3.5+}\left(5 d^{5.5}\right)$ partially filled delocalized bands ${ }^{39}, \mathrm{CuIr}_{2} \mathrm{~S}_{4}$ does not behave like a bandmetal, as evidenced by anomalous transport and spectroscopic signatures ${ }^{40,41}$. Despite early speculations to the contrary ${ }^{40-42}$, it was established that the structural dimers disappear on warming through the transition on all length scales, leaving the mystery of poor metallicity unresolved ${ }^{39}$. Curiously, the isostructural and isoelectronic sister compound, $\mathrm{CuIr}_{2} \mathrm{Se}_{4}$, has an order of magnitude higher conductivity and no MIT down to $0.5 \mathrm{~K}^{40}$, which is also difficult to rationalize within the current understanding of these systems.

In our high-sensitivity $\mathrm{x}$-ray pair distribution function (xPDF) analysis of the high-temperature metallic state of $\mathrm{CuIr}_{2} \mathrm{~S}_{4}$ we uncover a previously unobserved local symmetry lowering of the Ir pyrochlore sublattice, associated with an orbital liquid-like state that is present to the highest measured temperature. Through judicious chemical substitutions, we demonstrate that the effect is electronic and that it involves a symmetry lowering of the molecular orbitals, or Ir metal-metal bonds, on the pyrochlore sublattice. This is related to, but qualitatively different from, the dimer state observed in the insulating phase. It is Jahn-Teller like, in that the symmetry lowering breaks the degeneracy of partially filled Ir $d$ states, which results in orbital selectivity, with charges preferrentially selecting a subset of Ir-Ir metal-metal orbitals. At high temperature the selected orbitals do not order and are presumably fluctuating. This orbital liquid-like precursor state crystallizes upon approaching the Peierls-like MIT, testifying to the crucial role of orbital physics ${ }^{38}$.

Structural fingerprint of the ODL state. The PDF consists of peaks whose position is at interatomic distances in a material. It is therefore sensitive to any structural perturbation, because sharp single-valued PDF peaks in a high symmetry structure become broadened or multicomponent when the symmetry is lowered. In the low-temperature state of $\mathrm{CuIr}_{2} \mathrm{~S}_{4}$, long-range orbital and charge order results in $\mathrm{Ir}^{4+}-\mathrm{Ir}^{4+}$ pairs forming structural and magnetic dimers, which have been established crystallographically ${ }^{18}$. The Ir-Ir dimer pair distance is $\sim 0.5 \AA$ shorter than that of the Ir-Ir non-dimer pairs, creating two well-resolved peaks in the low-temperature PDF. In fact, the PDF dimer-peaks can be clearly seen by eye in the stack of PDFs shown as a function of temperature in Fig. 1a as a vertical ridge in the waterfall plot at $\approx 3 \AA$, labeled with the red arrow.

The dimers disappear in the average structure at $T_{\mathrm{MI}}{ }^{18}$, but they also disappear in the local structure, as first reported in ref. ${ }^{39}$, and which can be seen directly in the data in Fig. 1a. There is no dimer-liquid state at high temperature in $\mathrm{CuIr}_{2} \mathrm{~S}_{4}$, and the dimers themselves disappear at $T_{\mathrm{MI}}$, which rules out fluctuating dimers as the culprit behind the poor metallicity at high temperature.

We have approached the question of the anomalous metallic state by measuring a new, more complete and higher precision set of xPDF data from $\mathrm{CuIr}_{2} \mathrm{~S}_{4}$ (Fig. 1a), where we now focus on the high-temperature metallic state above $T_{\mathrm{MI}}$. The average crystal structure in this regime is cubic spinel, space-group $\mathrm{Fd} \overline{3} \mathrm{~m}$, in which the iridium ions make a pyrochlore sublattice that consists of a network of regular corner-shared tetrahedra illustrated in Fig. 1d. The high symmetry of this cubic structure results in an apparent sharpening of peaks in the PDF, as is evident in the waterfall plot in Fig. 1a, where PDF peak sharpening is observed on warming through $\mathrm{T}_{\mathrm{MI}}$ (normally $\mathrm{PDF}$ peaks broaden on warming due to increased atomic thermal motion). This occurs because the higher symmetry (cubic) phase has fewer PDF peaks 


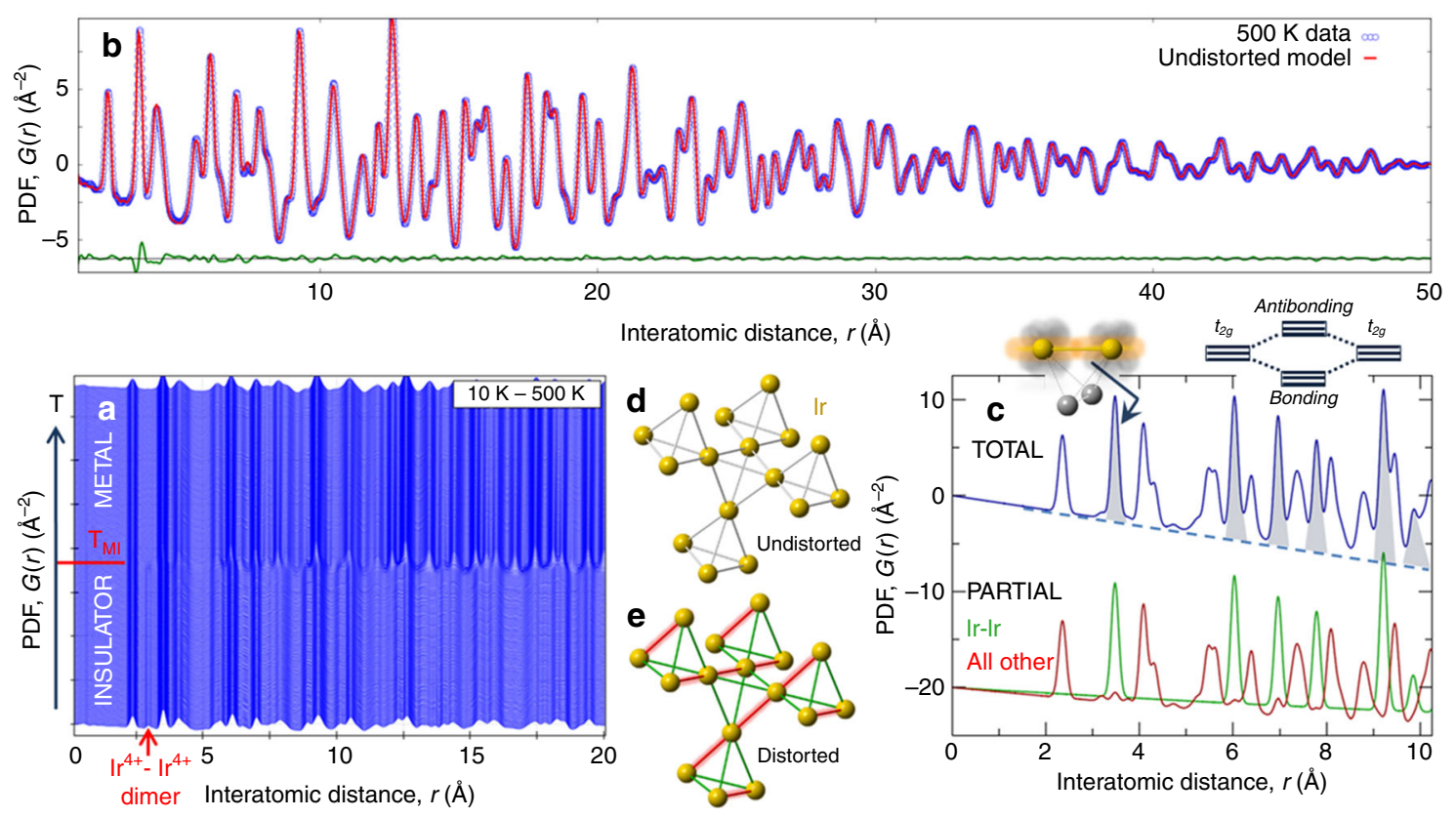

Fig. 1 Observation of high-temperature fluctuating ODL state in Culr $\mathrm{S}_{4}$. a Temperature waterfall stack of xPDFs measured on warming from $10 \mathrm{~K}$ (bottom) to $500 \mathrm{~K}$ (top) in $2 \mathrm{~K}$ increments. $T_{\mathrm{Ml}}$ is the MIT transition temperature (226 K). The dimer peak at $\sim 3 \AA$ (marked by arrow) is only seen in the insulating phase, and disappears above $T_{\mathrm{Ml}}$. b Fit of the undistorted cubic Fd $\overline{3} \mathrm{~m}$ model (red line) to the $500 \mathrm{~K}$ data (blue open circles) and their difference (green line, offset for clarity) unmask the footprint of the localized ODL state at $\sim 3.5 \AA$. c Simulated Fd $3 \bar{m}$ total xPDF of Culr $2 \mathrm{~S}_{4}$ (blue line), decomposed into Ir-Ir partial xPDF (green line) and its complement (red line). Shaded peaks in total xPDF are sensitive to $t_{2 g}$ orbital overlaps (sketched) and their spatial correlations. Inset: $t_{2 g}$-derived molecular orbitals discussed in the main text. d, e Section of Ir pyrochlore sublattice of corner-shared Ir 4 tetrahedra for undistorted (cubic) and distorted (tetragonal) spinel structure, respectively. The strongest $t_{2 g}$ orbital overlaps (e.g. $x y$ with $x y$, etc.) are along the chains formed by the tetrahedral edges of the Ir sublattice 38

than the lower symmetry (triclinic) phase. Indeed, fits of the cubic structure model to the high- $\mathrm{T}$ data result in excellent agreement (e.g., for $T=500 \mathrm{~K}, r_{\mathrm{w}}=5.1 \%$, Fig. $1 \mathrm{~b}$ ) Under normal circumstances this would be considered a highly satisfactory PDF fit. However, careful inspection of the residual curve in green in Fig. $1 \mathrm{~b}$ reveals a feature at around $3.5 \AA$, indicating a shift in intensity to higher $r$ in the data compared to the model.

The PDF peak centered at $3.5 \AA$ originates almost exclusively from the Ir-Ir nearest neighbor atomic pair on the pyrochlore sublattice, as shown in Fig. 1c. The total PDF consists of the weighted sum of partial PDFs between pairs of each type of atom, and Fig. 1c shows that the Ir-Ir partial-PDF contributes more than $95 \%$ of the signal to the $3.5 \AA$ peak in the total PDF. The residual signal therefore clearly originates from deviation of the structural geometry from the regular pyrochlore lattice implied by the cubic model. Importantly, maximal $t_{2 g}$ overlaps of the orbitals of the same type ( $x y$ with $x y, y z$ with $y z$, and $z x$ with $z x$ ) are precisely along the directions defined by the edges of the pyrochlore lattice $^{38}$, as sketched in the inset to Fig. 1c, implying that the orbital sector is involved.

Temperature evolution and characterization of the ODL state. To explore the temperature dependence, the same analysis is carried out on PDFs measured at temperatures up to $780 \mathrm{~K}$ and representative fits are shown in Fig. $2 \mathrm{a}-\mathrm{f}$. The result of the fitting for the $500 \mathrm{~K}$ dataset is reproduced in Fig. 2a over the entire $r$ range, and then on a narrower $r$-scale in Fig. $2 c$, with the residual signal highlighted. The same signal in the residual is also evident at $232 \mathrm{~K}, 300 \mathrm{~K}$ and in the $780 \mathrm{~K}$ data (Fig. $2 \mathrm{~d}-\mathrm{f}$, respectively). The $232 \mathrm{~K}$ dataset is from immediately above the MI transition temperature (226 K on warming).

To explore the structural origin of this residual signal we utilized structural models that allowed for distortions to the pyrochlore sublattice, and focused on a model in the $I 4_{1} /$ amd space group that was implicated in the early studies of the lowtemperature phase $^{33}$. In particular, the tetragonal distortion lowers the symmetry of the regular $\operatorname{Ir}_{4}$ pyrochlore tetrahedra, yielding 2 short and 4 long Ir-pair distances (Fig. 1e). Comparison of PDFs computed from the distorted and undistorted models, as seen in Fig. $2 \mathrm{~g}$, results in a difference curve that qualitatively reproduces the residual signal observed at $3.5 \AA$, Fig. 1b, when fitting with an undistorted cubic model. However, the tetragonal distortion leads to additional features in the PDF which are not seen in the measured data, suggesting that the tetragonal distortion is not appropriate at all length scales. Here we take advantage of the real-space nature of the PDF, and fit a tetragonal model over the narrow range $1.5<r<6 \AA$. This removes the residual signal at $3.5 \AA$ (Fig. $2 \mathrm{~h}$ ) and introduces only a single additional refinement parameter (tetragonal axis). The resulting fit produced a tetragonal distortion of $0.08(1) \AA$, which corresponds to long Ir-Ir bonds of $3.52 \AA$ and short Ir-Ir bonds of $3.44 \AA$.

This disparity in symmetry paints a clear picture where $\mathrm{Ir}_{4}$ tetrahedra, each with a local tetragonal distortion, are oriented in a disordered manner, such that individual distortions do not accumulate over long length scales, but rather average to a cubic symmetry. This is consistent with the observation that the low- $r$ portion of the PDF can be reproduced well only when using a tetragonally distorted model, but that this model fails to reproduce higher- $r$ features. Notably, the magnitude of the structural distortion at high temperature is $7 \times$ smaller than the distortion corresponding to the dimer state.

The correlation length of the ordering of such distorted tetrahedra can in principle be extracted from the PDF. Inspection of the residual curve in the $232 \mathrm{~K}$ data (Fig. 2b) suggests that at lower temperatures, though still above $T_{\mathrm{MI}}$, the fit of the cubic 

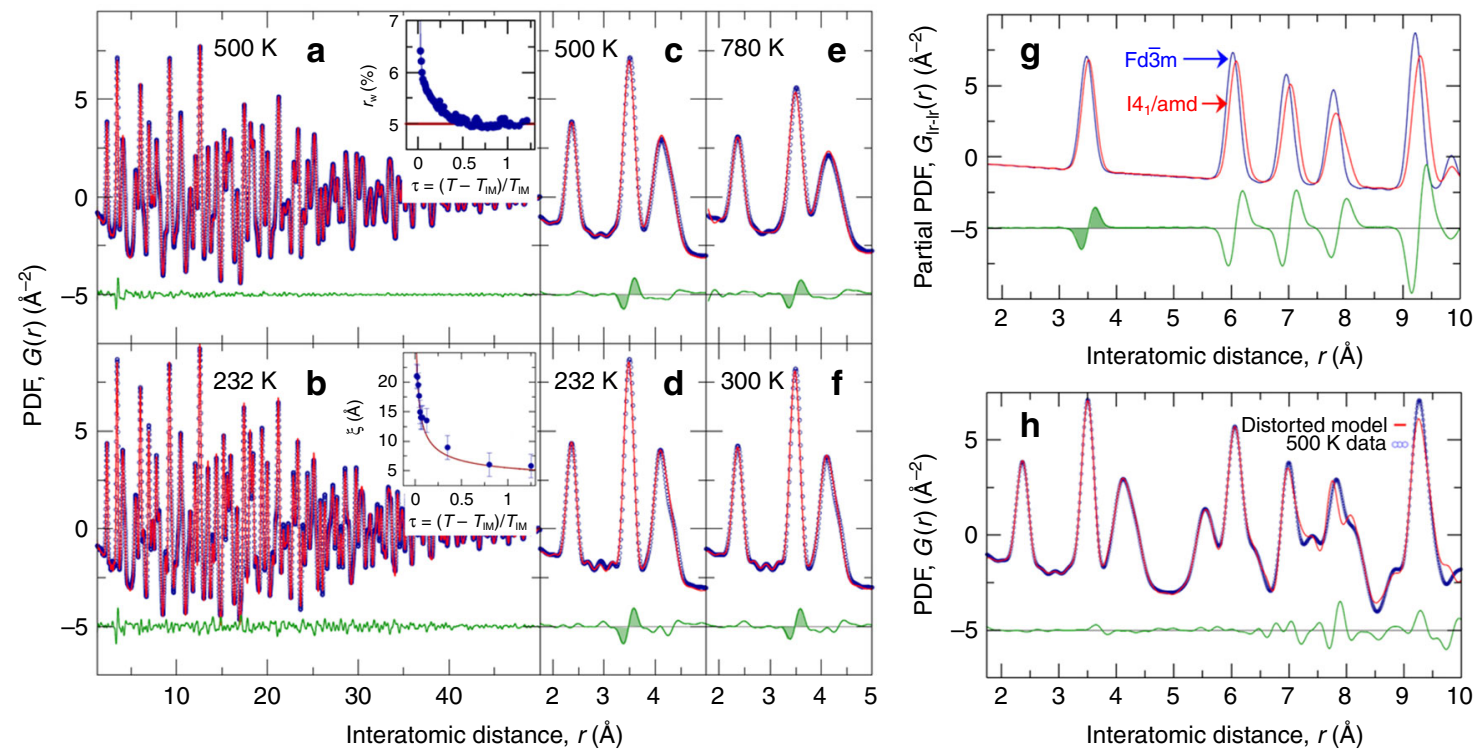

Fig. 2 Temperature evolution and character of the ODL state associated distortion. a-f Fit of the cubic Fd $\overline{3} m$ model (red line) to the Culr ${ }_{2} \mathrm{~S}_{4}$ data at various temperatures as indicated (blue open circles). Difference curve between the data and the model (green line) is offset for clarity in all cases. $\mathbf{g}$ Simulated Ir-Ir partial xPDFs for undistorted cubic Fd $3 \bar{m}$ (red line) and distorted tetragonal 141 /amd (blue line) structures, with the associated difference between the tetragonal and cubic models (green line) underneath, offset for clarity. $\mathbf{h}$ Short range $1.5<r<6 \AA$ fit of the distorted tetragonal model (red line) to the $500 \mathrm{~K}$ data (blue open circles), with associated difference curve underneath. The model and residual curves for $r>6 \AA$ represent a result of extending the calculation range without altering the fitted parameters. Insets to $\mathbf{a}, \mathbf{b}$ show evolution with reduced temperature of the cubic model fit residual, and the estimated local ODL distortion correlation length, respectively. Solid lines are guides to the eye

model is worse than at higher temperature and that the residual signal extends over a wider-range of $r$, up to $40 \AA$. This would be the case if the short bonds were beginning to short-range order with some correlation length. The temperature dependence of the correlation length, $\xi$, can be estimated using previously reported protocols ${ }^{43}$ and further described in Methods Section. The result is shown in the inset to Fig. 2b. The correlation length is $6 \AA$ at high temperature, but smoothly increases to $20 \AA$ as the MI transition is approached. This divergent behavior is mimicked if we consider the cubic model fit residual, $r_{\mathrm{w}}$, as a function of temperature (inset to Fig. 2a).

The symmetry breaking implies a breaking of the degeneracy of orbitals $^{42}$, which we refer to as an orbital-degeneracy-lifted state, dubbed ODL, on at least some of the Ir tetrahedra. The charge selects and preferrentially enters the lower energy orbitals, which may fluctuate among all the possible edges of the pyrochlore tetrahedra at high temperature (Fig. 3h). Such a phenomenon could be caused by various driving forces, including Jahn-Teller effects, covalency, or spin-orbit coupling 44,45 , and we do not speculate on the origin yet. However, we note that orbital selectivity impacts phenomena in diverse systems from $\mathrm{VO}_{2}{ }^{1}$, $\mathrm{K}_{2} \mathrm{Cr}_{8} \mathrm{O}_{16}{ }^{15}$, and $\mathrm{Sr}_{2-x} \mathrm{Ca}_{x} \mathrm{RuO}_{4}{ }^{46}$, to $\mathrm{FeSe}^{21,22,47}$, but the persistence of orbital selectivity to such high temperatures in a disordered orbital liquid state has not been widely observed. Our data show that the structural and metal-insulator transition on cooling corresponds not to the formation of an orbitally ordered state, but to the phase coherence and resulting long-range ordering of the pre-formed ODL objects.

Electronic manipulation of the ODL state. We now establish an electronic driving force for this ODL effect. Iridium takes on a nominal $5 d^{5.5}$ average electron configuration. In the cubic pyrochlore lattice the $t_{2 \mathrm{~g}}$ orbitals are well separated in energy from the $e_{\mathrm{g}}$ orbitals due to crystal field effects, and the $t_{2 \mathrm{~g}}$ orbital of one Ir points directly towards the neighboring $\mathrm{Ir}$ ion ${ }^{38,45}$. The large spatial extent of the $5 d$-states suggests a significant overlap of these orbitals and considerable covalency 45 , though this is not required for the discussion. We could then consider the orbital selectivity ${ }^{17}$, to happen on a basis of molecular orbitals ${ }^{48}$ (inset to Fig. 1c). In this case there would be a symmetry breaking into short and long edges on the pyrochlore tetrahedra depending on the electron filling of the molecular orbital but the incomplete filling of the $t_{2 \mathrm{~g}}$ manifold provides a Jahn-Teller-like driving force for the distortion that lifts the orbital degeneracy. Each Ir has six neighbors to choose from and randomize orbital selectivity. These concepts are illustrated in Fig. 3g, h top.

Altering the charge state of the Ir ions offers a method by which we can test this hypothesis. We have done this by doping $\mathrm{Zn}^{2+}$ on the $\mathrm{Cu}^{1+}$ site. The zinc doping increases the electron count in the Ir sublattice, without significantly disrupting the lattice ${ }^{49}$. The details are provided in Supplementary Note 1 and Supplementary Fig. 1, and the results are summarized in Fig. 3. $\mathrm{Zn}$ doping increases the electron count in an anti-bonding ODL state, marked with an asterisk in Fig. $3 \mathrm{~g}$. If the observed structural distortion is driven by a local Jahn-Teller effect, the feature in the residual should diminish with increasing $\mathrm{Zn}$ content as doping electrons in an anti-bonding band destabilizes the ODL state. This is exactly what is observed (Fig. 3c, d), establishing the electronic driving force for the effect.

We also consider the substitution of chromium on the iridium sublattice. Chromium is a small ion and introduces a compressive chemical pressure. It also introduces quenched defects into the Ir sublattice, disrupting the ability of Ir orbitals to order over long range at low temperature and suppressing the low-temperature orbital order state ${ }^{43}$. Fits of the cubic model to two of the $\mathrm{Cr}$ doped data-sets are shown in Fig. $3 e, f$. They clearly show that the signal in the residual at $3.5 \AA$ remains robustly up to $x=8 \%$, and indeed is stronger than in the $\mathrm{CuIr}_{2} \mathrm{~S}_{4}$ endmember, despite the absense of a symmetry-broken ground state. The compressive chemical pressure has the effect of stabilizing the ODL state, possibly due to an increasing $\operatorname{Ir}-\operatorname{Ir} t_{2 g}$ orbital overlap, suggesting 

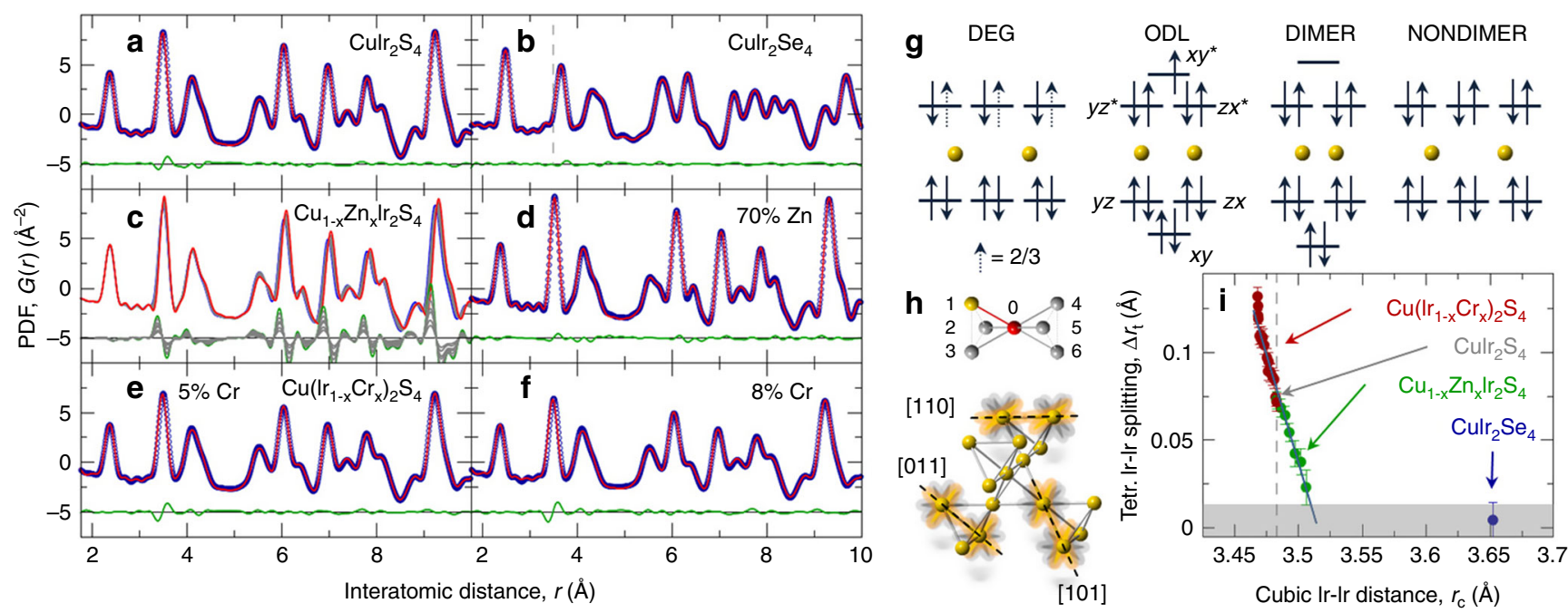

Fig. 3 Manipulation of the ODL state. a Fit of the cubic model (red line) to the $300 \mathrm{~K} \mathrm{Culr}{ }_{2} \mathrm{~S}_{4}$ data (blue open circles). $\mathbf{b}$ Fit of the cubic model (red line) to the $300 \mathrm{~K} \mathrm{Culr}_{2} \mathrm{Se}_{4}$ data (blue open circles). c Compositional stack of $300 \mathrm{~K}$ data for $\mathrm{Zn}$-substituted $\mathrm{Culr}_{2} \mathrm{~S}_{4}$ with $\mathrm{Zn}$ content ranging from $0 \%$ (blue line) to $70 \%$ (red line) in $10 \%$ increments (gray lines). The differences between the $\mathrm{Culr}_{2} \mathrm{~S}_{4}$ parent and all other datasets are stacked underneath, offset for clarity. The largest difference between the $0 \% \mathrm{Zn}$ and $70 \% \mathrm{Zn}$ datasets is shown in green, other differences in gray, evolving uniformly with $\mathrm{Zn}$ content. d Fit of the cubic model (red line) to the $300 \mathrm{~K} 70 \% \mathrm{Zn}$-substituted $\mathrm{Culr}_{2} \mathrm{~S}_{4}$ data (blue open circles). e, $\mathbf{f}$ Fit of the undistorted cubic model (red lines) to the $300 \mathrm{~K} 5 \%$ and $8 \% \mathrm{Cr}$-substituted $\mathrm{Culr}_{2} \mathrm{~S}_{4}$ data (blue open circles), respectively. $\mathbf{g}$ Molecular-orbital (MO) view, from left to right, of degenerate $\mathrm{MO}$, degeneracy-lifted $\mathrm{MO}$, dimerized, and non-dimerized Ir-Ir contacts. In the legend, DEG $\left(\mid \mathrm{r}^{3.5+}\right)$, ODL $\left(\mathrm{Ir}^{3.5+}\right)$, DIMER (Ir $\left.{ }^{4+}\right)$, and NONDIMER $\left(\mathrm{Ir}^{3+}\right)$. h Sketch of $[1,1,0]$-type Ir $t_{2 g}$ overlaps (bottom) and six choices for each Ir to form an ODL state (top). i Evolution of the ODL distortion, defined as the difference of the Ir-Ir nearest neighbor distance on a pyrochlore lattice extracted from local tetragonal model, with the average Ir-Ir separation in the cubic structure. These are extracted from fits to $300 \mathrm{~K}$ data of $\mathrm{Cr}$-substituted (red circles, $0<x<0.6$ ) and $\mathrm{Zn}$-substituted (green circles, $0<x<0.7$ ) samples, as well as pure $\mathrm{Culr}_{2} \mathrm{~S}_{4}$ (gray circle) and $\mathrm{Culr}_{2} \mathrm{Se}_{4}$ (blue circle). Error bars represent estimated standard uncertainties on the refined parameters. Gray shaded region marks $2 \sigma$ uncertainty for detecting small tetragonal distortions by the approach utilized here. $\ln$ Culr $_{2} \mathrm{~S}_{4}$ there are $0.5 t_{2 g}$ holes per $\operatorname{Ir}$ (one hole per pair) ${ }^{38}$. Vertical gray dashed lines in $\mathbf{b}$, i refer to $\mathrm{Culr}_{2} \mathrm{~S}_{4}$

that the symmetry lowering is among molecular orbitals rather than atomic $d$-states.

Finally, we consider the sister compound $\mathrm{CuIr}_{2} \mathrm{Se}_{4}$. In this case the ODL signature in the fit residual is absent, Fig. $3 \mathrm{~b}$. In $\mathrm{CuIr}_{2} \mathrm{Se}_{4}$ the electron counting arguments are the same as in $\mathrm{CuIr}_{2} \mathrm{~S}_{4}$, with the Ir $t_{2 \mathrm{~g}}$ states being at the Fermi-level, implying similar physics. However, the Se ion is considerably larger than the S ion, which would result in a larger inter-Ir distance and reduced Ir-Ir $t_{2 \mathrm{~g}}$ orbital overlap in the case of $\mathrm{CuIr}_{2} \mathrm{Se}_{4}$. In a traditional site-centered Jahn-Teller picture this would not affect the driving force, indeed it may even make the Jahn-Teller distortion larger by lowering the elastic stiffness of the material. However, if covalency between neighboring Ir ions is important, as the data suggest is the case here, we speculate that the reduced orbital overlap of the Ir $t_{2 g}$ orbitals would reduce the splitting of the bonding and anti-bonding orbitals, which would reduce the driving force for the distortion. Indeed, we see an anticorrelation between the size of the tetragonal distortion and the magnitude of the average Ir-Ir distance as we vary the composition by $\mathrm{Zn}$ doping and $\mathrm{Cr}$ doping (Fig. 3i), consistent with a stronger $t_{2 \mathrm{~g}}$ orbital overlap strengthening the ODL effect. The Ir-Ir distance in $\mathrm{CuIr}_{2} \mathrm{Se}_{4}$ is also shown in Fig. 3i, and it is much higher. Notably, we do not observe any tetragonal distortion even in the local structure in $\mathrm{CuIr}_{2} \mathrm{Se}_{4}$, again supporting the importance of the Ir-Ir covalency in the ODL mechanism in this case. The importance of covalency would also suggest that the orbitaldegeneracy lifting may be stabilized by pressure, since pressure would increase the overlap of neighboring $t_{2 g}$ orbitals. Indeed, under pressure $\mathrm{CuIr}_{2} \mathrm{Se}_{4}$ does undergo a metal-insulator transition as reflected in transport measurements ${ }^{50}$. This observation also provides an explanation of why the MIT temperature increases with pressure in $\mathrm{CuIr}_{2} \mathrm{~S}_{4}{ }^{51}$, a trend opposite to that seen in conventional Fermi-surface nesting driven charge-densitywave systems.

We have shown that in $\mathrm{CuIr}_{2} \mathrm{~S}_{4}$ the origin of the orbitaldegeneracy lifting is a local symmetry lowering of Ir-Ir $t_{2 \mathrm{~g}}$ molecular orbitals. We briefly note here that $5 d$ ions such as Ir are also susceptible to an orbital-degeneracy lifting due to spin-orbit coupling. ODL due to spin-orbit effects are prevalent in predominately $\mathrm{Ir}^{4+}$ oxides such as $\mathrm{Sr}_{2} \mathrm{IrO}_{4}{ }^{52,53}$ resulting in an isospin-1/2 relativistic Mott insulating ground-state. We can speculate that in the current $\operatorname{Ir}^{3.5+}$ case, and with sufficient orbital overlap, valence electron itinerancy may dominate over the atomic picture that is the basis for the SOC $^{38}$. Learning what factors determine whether SOC or Jahn-Teller effects govern the orbital-degeneracy lifting will be an interesting line of inquiry.

Implications. The characterization of the high-temperature state of $\mathrm{CuIr}_{2} \mathrm{~S}_{4}$ as being an ODL state, made up of local symmetrybroken objects stabilized by orbital-degeneracy lifting, presents a potential unifying framework and a new lens through which to view multiple material systems. Calculations that derive from the crystal structure, such as density functional theory calculations, should therefore be modified to account for the very different (tenths of an angstrom) bond-lengths that may be present in the material ${ }^{54}$. This would not be necessary if the ground state consisted simply of ODL objects whose orbitals become ordered over long-range at low temperature. However, often the ground state is quite different from this, as in $\mathrm{CuIr}_{2} \mathrm{~S}_{4}$ where it consists of charge order, with structural and magnetic dimers, none of which persist above the MIT and into the ODL state ${ }^{39}$. Likewise, if we view the insulating polaronic state in the colossal magnetoresistant $30 \%$ doped $\mathrm{La}_{1-x} \mathrm{Ca}_{x} \mathrm{MnO}_{3}$ manganites ${ }^{55}$ as an ODL state, the low-temperature ground state has been shown to be absent 
structural distortions ${ }^{19}$ and is a non-ODL state. In the $\mathrm{CuIr}_{2} \mathrm{~S}_{4}$ case long-range ordering may be suppressed due to the geometric frustration of disordering a short Ir-Ir bond over the six edges of the tetrahedron in the pyrochlore lattice, a problem that maps onto the Pauling ice rules ${ }^{56,57}$. The high-temperature ODL state is in general not just a disordered form of the ground state and needs to be studied independently and in its own right. This is not straightforward, requiring probes of local structure and the local electronic system. Because the objects are local and fluctuating, they are not observable in the crystal structure. The ODL objects are also, in general, fluctuating in the disordered ODL state thus requiring probes that are also faster than any fluctuation dynamics. However, because the nature of the ODL formation is electronic, we expect that optically pumped ultrafast timeresolved measurements of local structure should be a powerful approach to investigate $\mathrm{ODL}^{58,59}$.

\section{Methods}

Sample preparation and characterization. Polycrystalline samples of $\mathrm{CuIr}_{2} \mathrm{~S}_{4}$, $\mathrm{CuIr}_{2} \mathrm{Se}_{4},\left(\mathrm{Cu}_{1-x} \mathrm{Zn}_{x}\right) \mathrm{Ir}_{2} \mathrm{~S}_{4}$, and $\mathrm{Cu}\left(\mathrm{Ir}_{1-x} \mathrm{Cr}_{x}\right)_{2} \mathrm{~S}_{4}$ were prepared following standard solid state routes in sealed, evacuated quartz ampoules. Stoichiometric quantities of the metals and elemental sulfur or selenium were thoroughly mixed, pelletized, and sealed under vacuum. The ampoules were slowly heated to various temperatures in $650-1100^{\circ} \mathrm{C}$ range, as appropriate to targeted compositions, and held at these temperatures for several weeks with intermediate grinding and pressing. All products were found to be single phase based on laboratory $\mathrm{x}$-ray powder diffraction. Standard characterization of DC susceptibility and four-terminal resistivity of the samples were carried out in Quantum Design PPMS-9 and MPMS-XL5, and found to be in excellent agreement with other studies $18,33,35,40,49,60$.

PDF data collection and analysis. PDF data for $10 \mathrm{~K} \leq T \leq 780 \mathrm{~K}$ were obtained using standard protocols ${ }^{61}$ from synchrotron $\mathrm{x}$-ray total scattering experiments carried out at the 28-ID-2 x-ray powder diffraction (XPD) beamline of the National Synchrotron Light Source II at Brookhaven National Laboratory. The setup utilized a $67.7 \mathrm{keV}$ x-ray beam $(\lambda=0.183 \AA)$, a Perkin Elmer amorphous silicon detector, a closed cycle Cryoindustries of America helium refrigerator, and a gas flow reactor with flexible coil heater. Two dimensional (2D) diffraction data were collected in rapid acquisition mode ${ }^{62}$, with $60 \mathrm{~s}$ exposure time for each data set. The raw 2D data (collected on warming) were integrated and converted to intensity versus $Q$ using the software Fit $2 \mathrm{D}^{63}$, where $Q$ is the magnitude of the scattering vector. Data reduction and Sine Fourier transform of measured total scattering structure functions up to a momentum transfer of $Q_{\max }=25 \AA^{-1}$ was carried out using the PDFgetX $3^{64}$ program. PDF structure refinements and simulations were carried out using the PDFgui program suite ${ }^{65}$.

Correlation length estimate was based on a protocol utilizing a 4- $\AA$ wide box car window integration of the residual difference between the data and the cubic $\mathrm{Fd} \overline{3} \mathrm{~m}$ model. $\xi$ is then defined as the $r$ value at which the integral drops by a factor of 2 from its low- $r$ limit, with uncertainty of the estimate conservatively set to half of the window size, similar to correlation length estimates carried out in past PDF studies ${ }^{43,66}$

\section{Data availability}

The data supporting the findings of this study are within the Article and its Supplementary Information files and are available from the corresponding author upon request.

Received: 21 March 2019 Accepted: 9 July 2019

Published online: 13 August 2019

\section{References}

1. Aetukuri, N. B. et al. Control of the metal-insulator transition in vanadium dioxide by modifying orbital occupancy. Nat. Phys. 9, 661-666 (2013).

2. Tian, Z. et al. Field-induced quantum metal-insulator transition in the pyrochlore iridate $\mathrm{Nd}_{2} \mathrm{Ir}_{2} \mathrm{O}_{7}$. Nat. Phys. 12, 134-138 (2016).

3. Liang, T. et al. Orthogonal magnetization and symmetry breaking in pyrochlore iridate $\mathrm{Eu}_{2} \mathrm{Ir}_{2} \mathrm{O}_{7}$. Nat. Phys. 13, 599-603 (2017).

4. Achkar, A. J. et al. Orbital symmetry of charge-density-wave order in $\mathrm{La}_{1.875} \mathrm{Ba}_{0.125} \mathrm{CuO}_{4}$ and $\mathrm{YBa}_{2} \mathrm{Cu}_{3} \mathrm{O}_{6.67}$. Nat. Mater. 15, 616-620 (2016).

5. Savitzky, B. H. et al. Bending and breaking of stripes in a charge ordered manganite. Nat. Commun. 8, 1883 (2017).
6. Zorko, A., Adamopoulos, O., Komelj, M., Arčon, D. \& Lappas, A. Frustrationinduced nanometre-scale inhomogeneity in a triangular antiferromagnet. Nat. Commun. 5, 3222 (2014).

7. Glasbrenner, J. K. et al. Effect of magnetic frustration on nematicity and superconductivity in iron chalcogenides. Nat. Phys. 11, 953-958 (2015).

8. Borisenko, S. V. et al. Pseudogap and charge density waves in two dimensions. Phys. Rev. Lett. 100, 196402 (2008).

9. Keimer, B., Kivelson, S. A., Norman, M. R., Uchida, S. \& Zaanen, J. From quantum matter to high-temperature superconductivity in copper oxides. Nature 518, 179-186 (2015).

10. Wang, F., Kivelson, S. A. \& Lee, D.-H. Nematicity and quantum paramagnetism in FeSe. Nat. Phys. 11, 959-963 (2015).

11. Chuang, Y., Gromko, A., Dessau, D., Kimura, T. \& Tokura, Y. Fermi surface nesting and nanoscale fluctuating charge/orbital ordering in colossal magnetoresistive oxides. Science 292, 1509-1513 (2001).

12. Johannes, M. D. \& Mazin, I. I. Fermi surface nesting and the origin of charge density waves in metals. Phys. Rev. B 77, 165135 (2008).

13. Terashima, K. et al. Fermi surface nesting induced strong pairing in ironbased superconductors. Proc. Natl Acad. Sci. USA 106, 7330-7333 (2009).

14. Lee, P. A., Rice, T. M. \& Anderson, P. W. Fluctuation effects at a peierls transition. Phys. Rev. Lett. 31, 462-465 (1973).

15. Bhobe, P. A. et al. Electronic structure evolution across the Peierls metalinsulator transition in a correlated ferromagnet. Phys. Rev. X 5, 041004 (2015).

16. Huang, H. Y. et al. Jahn-Teller distortion driven magnetic polarons in magnetite. Nat. Commun. 8, 15929 (2017).

17. Khomskii, D. I. Transition Metal Compounds (Cambridge University Press, Cambridge, UK, 2014).

18. Radaelli, P. G. et al. Formation of isomorphic $\mathrm{Ir}^{3+}$ and $\mathrm{Ir}^{4+}$ octamers and spin dimerization in the spinel $\mathrm{CuIr}_{2} \mathrm{~S}_{4}$. Nature 416, 155-158 (2002).

19. Billinge, S. J. L., DiFrancesco, R. G., Kwei, G. H., Neumeier, J. J. \& Thompson, J. D. Direct observation of lattice polaron formation in the local structure of $\mathrm{La}_{1-x} \mathrm{Ca}_{x} \mathrm{MnO}_{3}$. Phys. Rev. Lett. 77, 715-718 (1996).

20. Billinge, S. J. L. \& Duxbury, P. M. Structural compliance, misfit strain and stripe nanostructures in cuprate superconductors. Phys. Rev. B 66, 064529 (2002).

21. Sprau, P. O. et al. Discovery of orbital-selective Cooper pairing in FeSe. Science 357, 75-80 (2017)

22. Kostin, A. et al. Imaging orbital-selective quasiparticles in the Hund's metal state of FeSe. Nat. Mater. 17, 869-874 (2018).

23. Massee, F. et al. Bilayer manganites reveal polarons in the midst of a metallic breakdown. Nat. Phys. 7, 978-982 (2011).

24. Panopoulos, $\mathrm{N}$. et al. Polaron freezing and the quantum liquid-crystal phase in the ferromagnetic metallic $\mathrm{La}_{0.67} \mathrm{Ca}_{0.33} \mathrm{MnO}_{3}$. npj Quantum Mater. 3, 20 (2018).

25. Scagnoli, V. et al. Observation of orbital currents in $\mathrm{CuO}$. Science 332, 696-698 (2011).

26. Wen, Y.-C. et al. Gap opening and orbital modification of superconducting FeSe above the structural distortion. Phys. Rev. Lett. 108, 267002 (2012).

27. Kasahara, S. et al. Electronic nematicity above the structural and superconducting transition in $\mathrm{BaFe}_{2}\left(\mathrm{As}_{1-x} \mathrm{P}_{x}\right)_{2}$. Nature 486, 382-385 (2012).

28. Baek, S.-H. et al. Orbital-driven nematicity in FeSe. Nat. Mater. 14, 210-214 (2015).

29. Frandsen, B. A. et al. Widespread orthorhombic fluctuations in the ( $\mathrm{Sr}, \mathrm{Na})$ $\mathrm{Fe}_{2} \mathrm{As}_{2}$ family of superconductors. Phys. Rev. B 98, 180505 (2018).

30. Radaelli, P. G. Orbital ordering in transition-metal spinels. New J. Phys. 7, 53 (2005).

31. Attfield, J. P. Orbital molecules in electronic materials. APL Mater. 3, 041510 (2015).

32. Rivas-Murias, B., Zhou, H. D., Rivas, J. \& Rivadulla, F. Rapidly fluctuating orbital occupancy above the orbital ordering transition in spin-gap compounds. Phys. Rev. B 83, 165131 (2011).

33. Furubayashi, T., Matsumoto, T., Hagino, T. \& Nagata, S. Structural and magnetic studies of metal-insulator transition in thiospinel $\mathrm{CuIr}_{2} \mathrm{~S}_{4}$. J. Phys. Soc. Jpn 63, 3333-3339 (1994)

34. Matsuno, J. et al. Photoemission study of the metal-insulator transition in cuir2s4. Phys. Rev. B 55, R15979 (1997).

35. Nagata, S. et al. Metal-insulator transition in the spinel-type $\mathrm{CuIr}_{2}\left(\mathrm{~S}_{1-x} \mathrm{Se}_{x}\right)_{4}$ system. Phys. Rev. B 58, 6844-6854 (1998).

36. Takubo, K. et al. X-ray photoemission study of $\mathrm{CuIr}_{2} \mathrm{~S}_{4}: \mathrm{Ir}^{3+}-\mathrm{Ir}^{4+}$ charge ordering and the effect of light illumination. Phys. Rev. Lett. 95, 246401 (2005).

37. Croft, M. et al. Metal-insulator transition in $\mathrm{CuIr}_{2} \mathrm{~S}_{4}$ : XAS results on the electronic structure. Phys. Rev. B 67, 201102 (2003).

38. Khomskii, D. I. \& Mizokawa, T. Orbitally induced Peierls state in spinels. Phys. Rev. Lett. 94, 156402 (2005).

39. Božin, E. S., Masadeh, A. S., Hor, Y. S., Mitchell, J. F. \& Billinge, S. J. L. Detailed mapping of the local $\mathrm{Ir}^{4+}$ dimers through the metal-insulator transitions of $\mathrm{CuIr}_{2} \mathrm{~S}_{4}$ thiospinel by $\mathrm{x}$-ray atomic pair distribution function measurements. Phys. Rev. Lett. 106, 045501 (2011). 
40. Burkov, A. T. et al. Anomalous resistivity and thermopower of the spinel-type compounds $\mathrm{CuIr}_{2} \mathrm{~S}_{4}$ and $\mathrm{CuIr}_{2} \mathrm{Se}_{4}$. Phys. Rev. B 61, 10049-10056 (2000).

41. Takubo, K., Mizokawa, T., Matsumoto, N. \& Nagata, S. In-gap state and effect of light illumination in $\mathrm{CuIr}_{2} \mathrm{~S}_{4}$ probed by photoemission spectroscopy. Phys. Rev. B 78, 245117 (2008).

42. Yagasaki, K. et al. Hopping conductivity in $\mathrm{CuIr}_{2} \mathrm{~S}_{4}$ spinel compound: I. empirical model for electronic configuration and mechanism of metalinsulator transition. J. Phys. Soc. Jpn 75, 074706 (2006).

43. Božin, E. S. et al. $\mathrm{Cu}\left(\operatorname{Ir}_{1-x} \mathrm{Cr}_{x}\right)_{2} \mathrm{~S}_{4}$ : a model system for studying nanoscale phase coexistence at the metal-insulator transition. Sci. Rep. 4, 4081 (2014).

44. Streltsov, S. V. \& Khomskii, D. I. Covalent bonds against magnetism in transition metal compounds. Proc. Natl Acad. Sci. USA 113, 10491-10496 (2016).

45. Streltsov, S. V. \& Khomskii, D. I. Orbital physics in transition metal compounds: new trends. Phys. Uspekhi 60, 1121-1146 (2017).

46. de' Medici, L., Giovannetti, G. \& Capone, M. Selective Mott physics as a key to iron superconductors. Phys. Rev. Lett. 112, 177001 (2014).

47. Yu, R., Zhu, J.-X. \& Si, Q. Orbital selectivity enhanced by nematic order in FeSe. Phys. Rev. Lett. 121, 227003 (2018).

48. Lei, H., Yin, W.-G., Zhong, Z. \& Hosono, H. Structural, magnetic, and electrical properties of $\mathrm{Li}_{2} \mathrm{Ir}_{1-x} \mathrm{Ru}_{x} \mathrm{O}_{3}$. Phys. Rev. B 89, 020409 (2014).

49. Cao, G. et al. Suppression of metal-to-insulator transition and appearance of superconductivity in $\mathrm{Cu}_{1-x} \mathrm{Zn}_{x} \mathrm{Ir}_{2} \mathrm{~S}_{4}$. Phys. Rev. B 64, 214514 (2001).

50. Furubayashi, T. et al. Pressure induced metal-insulator transition of selenospinel CuIr $\mathrm{Se}_{4}$. J. Phys. Soc. Jpn 66, 1563-1564 (1997).

51. Ma, L. et al. Opposite pressure effects in the orbitally-induced Peierls phase transition systems $\mathrm{CuIr}_{2} \mathrm{~S}_{4}$ and $\mathrm{MgTi}_{2} \mathrm{O}_{4}$. Dalton Trans. 46, 6708-6714 (2017).

52. Martins, C., Aichhorn, M., Vaugier, L. \& Biermann, S. Reduced effective spinorbital degeneracy and spin-orbital ordering in paramagnetic transition-metal oxides: $\mathrm{Sr}_{2} \mathrm{IrO}_{4}$ versus $\mathrm{Sr}_{2} \mathrm{RhO}_{4}$. Phys. Rev. Lett. 107, 266404 (2011).

53. Kim, B. J. et al. Phase-sensitive observation of a spin-orbital Mott state in $\mathrm{Sr}_{2} \mathrm{IrO}_{4}$. Science 323, 1329-1332 (2009)

54. Varignon, J., Bibes, M. \& Zunger, A. Origin of band gaps in 3d perovskite oxides. Nat. Commun. 10, 1658 (2019)

55. Millis, A. J. Lattice effects in magnetoresistive manganese perovskites. Nature 392, 147-150 (1998).

56. Thygesen, P. M. et al. Orbital dimer model for the spin-glass state in $\mathrm{Y}_{2} \mathrm{Mo}_{2} \mathrm{O}_{7}$. Phys. Rev. Lett. 118, 067201 (2017).

57. Bramwell, S. T. \& Gingras, M. J. P. Spin ice state in frustrated magnetic pyrochlore materials. Science 294, 1495-1501 (2001).

58. Koch, R. et al. Room temperature local nematicity in FeSe superconductor. Preprint at https://arxiv.org/abs/1902.08732 (2019).

59. Konstantinova, T. et al. Photoinduced dynamics of nematic order parameter in FeSe. Phys. Rev. B 99, 180102 (2019).

60. Endoh, R., Awaka, J. \& Nagata, S. Ferromagnetism and the metal-insulator transition in the thiospinel $\mathrm{Cu}\left(\mathrm{Ir}_{1-x} \mathrm{Cr}_{x}\right)_{2} \mathrm{~S}_{4}$. Phys. Rev. B 68, 115106 (2003).

61. Egami, T. \& Billinge, S. J. L. Underneath The Bragg Peaks: Structural Analysis Of Complex Materials. 2nd edn (Elsevier, Amsterdam, 2012).

62. Chupas, P. J. et al. Rapid acquisition pair distribution function analysis (RAPDF). J. Appl. Crystallogr. 36, 1342-1347 (2003).

63. Hammersley, A. P., Svenson, S. O., Hanfland, M. \& Hauserman, D. Twodimensional detector software: from real detector to idealised image or twotheta scan. High Press. Res. 14, 235-248 (1996).

64. Juhás, P., Davis, T., Farrow, C. L. \& Billinge, S. J. L. PDFgetX3: a rapid and highly automatable program for processing powder diffraction data into total scattering pair distribution functions. J. Appl. Crystallogr. 46, 560-566 (2013).

65. Farrow, C. L. et al. PDFfit2 and PDFgui: computer programs for studying nanostructure in crystals. J. Phys. Condens. Mater. 19, 335219 (2007).

66. Qiu, X., Proffen, T., Mitchell, J. F. \& Billinge, S. J. L. Orbital correlations in the pseudocubic $O$ and rhombohedral $R$-phases of $\mathrm{LaMnO}_{3}$. Phys. Rev. Lett. 94, 177203 (2005).

\section{Acknowledgements}

Work at Brookhaven National Laboratory was supported by US DOE, Office of Science, Office of Basic Energy Sciences under contract DE-SC0012704. Work in the Materials Science Division of Argonne National Laboratory, was sponsored by the U.S. Department of Energy Office of Science, Basic Energy Sciences, Materials Science and Engineering Division. This research used 28-ID-2 beamline of the National Synchrotron Light Source II, a U.S. Department of Energy (DOE) Office of Science User Facility operated for the DOE Office of Science by Brookhaven National Laboratory.

\section{Author contributions}

E.S.B., J.F.M. and S.J.L.B. conceived and designed the research. C.P., H.C.L., J.F.M., Y.S.H., and H.Z. developed and carried out the synthesis and did material characterizations. E.S.B., R.J.K. and M.A. carried out PDF measurements and analysis. W.G.Y provided theoretical inputs. E.S.B., S.J.L.B. and J.F.M. wrote the manuscript with contributions from all the authors.

\section{Additional information}

Supplementary Information accompanies this paper at https://doi.org/10.1038/s41467 019-11372-w.

Competing interests: The authors declare no competing interests.

Reprints and permission information is available online at http://npg.nature.com/ reprintsandpermissions/

Peer review information: Nature Communications thanks the anonymous reviewers for their contribution to the peer review of this work.

Publisher's note: Springer Nature remains neutral with regard to jurisdictional claims in published maps and institutional affiliations.

Open Access This article is licensed under a Creative Commons Attribution 4.0 International License, which permits use, sharing, adaptation, distribution and reproduction in any medium or format, as long as you give appropriate credit to the original author(s) and the source, provide a link to the Creative Commons license, and indicate if changes were made. The images or other third party material in this article are included in the article's Creative Commons license, unless indicated otherwise in a credit line to the material. If material is not included in the article's Creative Commons license and your intended use is not permitted by statutory regulation or exceeds the permitted use, you will need to obtain permission directly from the copyright holder. To view a copy of this license, visit http://creativecommons.org/ licenses/by/4.0/

(C) The Author(s) 2019 